\title{
Amorphous silicate nanoparticles with controlled Fe-Mg pyroxene compositions
}

\author{
Stephen P. Thompson ${ }^{\mathrm{a}, 1, *}$, Karine Demyk ${ }^{\mathrm{b}, \mathrm{c}}$, Sarah J. Day ${ }^{\mathrm{a}, \mathrm{e}}$, Aneurin \\ Evans $^{\mathrm{e}}$, Hugues Leroux ${ }^{\mathrm{d}}$, Christophe Depecker ${ }^{\mathrm{d}}$, Julia E. Parker ${ }^{\mathrm{a}}$, Leigh \\ Connor $^{\mathrm{a}}$, Heribert Wilhelm ${ }^{\mathrm{a}}$, Giannantonio Cibin ${ }^{\mathrm{a}}$ \\ ${ }^{a}$ Diamond Light Source, Harwell Science and Innovation Campus, Chilton, Didcot, \\ Oxfordshire OX11 ODE, UK \\ ${ }^{b}$ Université de Toulouse; UPS-OMP; IRAP; Toulouse, France \\ ${ }^{c} I R A P$ - Institut de Recherche en Astrophysique et Planétologie, 9 avenue du Colonel \\ Roche, BP 44346, F-31028 Toulouse cedex 4, France \\ ${ }^{d}$ Unité Matériaux et Transformations, Université Lille 1, 59655 Villeneuve d'Ascq, \\ France \\ ${ }^{e}$ Astrophysics Group, Keele University, Keele, Staffs ST5 5BG, UK
}

\section{Abstract}

The production of amorphous pyroxene nanoparticles $(\sim 20 \mathrm{~nm})$ with controlled Fe-Mg content is described. Homogenous particle compositions closely matching required target stoichiometries are obtained by drying a precursor gel under high vacuum conditions. The silicate nature of the particles is characterised using TEM, synchrotron radiation and FTIR. No oxide phase separation occurs, even at high Fe concentration. Structural domains exist within the nanoparticles that are typically ten times smaller than the physical particle size consistent with either a core-shell, or random network with multiple embedded domains, particle structure. Thermal annealing below the crystallisation temperature allows the ordered domain size to be further

\footnotetext{
* Corresponding author

Email address: stephen.thompson@diamond.ac.uk (Stephen P. Thompson)

${ }^{1}$ Tel: $++44(0) 1235778546$
} 
reduced by a factor of $\sim 2$.

Keywords: Iron and Magnesium silicate, Nanoparticles, Sol-gel, Amorphous material

\section{Introduction}

Nanoparticles are an important class of materials due to the enhancement, over their bulk equivalents, of properties such as optical activity, cation exchange capacity, surface area, chemical reactivity and adsorptivity; and have made significant contributions to a wide range of engineering, technology, biomedical, manufacturing, communications and structural applications as well as being of interest to academic research.

Crystalline nanoparticles have a large fraction of their atoms located on their surface and the presence of structural disorder near the surface results in functional properties significantly different to the bulk crystalline material [1]. Amorphous nanoparticles (ANPs) on the other hand are generally believed [2] to have either a two-part core-shell structure, whereby an amorphous core is surrounded by a more porous surface shell region containing a large amount of structural defects; or to have a layered structure comprising high and low density radial layers. Because of these structural differences ANPs often have very different properties and applications compared to crystalline nanoparticles [2]. Fe and Fe-oxide ANPs, for example, exhibit unique magnetic, catalytic, optical and sorption properties relevant to advanced technological applications [3]. Unfortunately, Fe's preference for forming oxide phases under atmospheric, or similarly oxidising conditions, 
means that its controlled incorporation into other materials such as silicate is problematic and complicated by its ability to act as both network former and network modifier.

However, Fe-Mg silicate ANPs of pyroxene (and related) silicate compositions are present in the Earth's upper atmosphere $[4,5]$, having formed by the recondensation of $\mathrm{Fe}, \mathrm{Mg}$ and $\mathrm{Si}$ oxide species produced by the ongoing ablation of in-falling meteoroids [6]. These particles are present in sufficient quantities $\left(\sim 1000\right.$ 's per $\left.\mathrm{cm}^{3}[4]\right)$ to play a significant role in a number of atmospheric processes, including cloud nucleation and removal of $\mathrm{H}_{2} \mathrm{SO}_{4}$ from the gas phase at high altitude. Laboratory dissolution studies using analogue ANPs provide the physical constraints necessary to assess their environmental impact via modelling [7]. Similarly in astrophysics, Mg-rich pyroxene ANPs condense as dust particles (along with other solid species) in the circumstellar regions surrounding evolved stars and are ubiquitous throughout space. However, the fate of elemental Fe is a significant unsolved problem in astrophysics [8]. Observations show it is highly depleted from the interstellar gas phase and must therefore reside somewhere within the solid phase population. The absence of observable IR features attributable to pure-phase metallic Fe or Fe-oxide phases means that current theories assume Fe is somehow incorporated within the silicate ANP dust, even though fits to observational IR spectra favour Mg-rich, Fe-poor compositions. Since thermodynamics cannot predict the $\mathrm{Mg} / \mathrm{Fe}$ distribution between amorphous phases, laboratory simulation using analogue ANP materials with variable Fe content represents the only means of investigation. However, the laboratory approach is challenging as the properties of amorphous nanomaterials (and 
specifically nanosilicates) remain poorly understood and reliable, controlled production methods capable of yielding large volumes suitable for multiple experiments are largely absent $[2,9]$.

Early attempts at synthesising amorphous Fe-bearing silicates met with only limited success and were generally produced as one-offs [10, 11], while more systematic approaches applied glass preparation methods that involved melt quenching [12]. However, spectroscopic behaviour and physical homogeneity were strongly influenced by the quenching method rather than composition, as was the Fe oxidation state due to the melting/quenching taking place in air. Furthermore, moderately high Fe content resulted in phase separation and varying crystallisation [13] even in a reducing atmosphere [14]. Attempts to produce silicate ANPs via vapour phase condensation in controlled $\mathrm{Mg}-\mathrm{Fe}-\mathrm{SiO}-\mathrm{H}_{2}-\mathrm{O}_{2}$ atmospheres produced only pure $\mathrm{Mg}$ or pure Fe silicates whose compositions corresponded to the metastable eutectics in the $\mathrm{MgO}-\mathrm{SiO}_{2}$ and $\mathrm{FeO} / \mathrm{Fe}_{2} \mathrm{O}_{3}-\mathrm{SiO}_{2}$ binary phase diagrams [15]. Other researchers found mixed Fe-Mg ANPs could be produced by the laser ablation of mineral targets. However, differences in the optical constants, thermal properties and the melting temperatures of different oxides result in variable evaporation rates, which adversely affect compositional homogeneity [16]. Of more relevance to the present work was an attempt to synthesise amorphous silicates with chondritic composition (i.e. $\mathrm{Na}_{2} \mathrm{O}-\mathrm{MgO}-\mathrm{Al}_{2} \mathrm{O}_{3}-\mathrm{SiO}_{2}-\mathrm{CaO}-\mathrm{FeO}-$ $\mathrm{NiO}$ after the composition of $\mathrm{C} 1$ meteorites [17]) using a sol-gel approach. However, the final product consisted of an amorphous matrix containing separated crystallites of magnetite and ferrihydrite $[18,8]$.

In all of the above syntheses, the degree to which Fe is incorporated 
into the silicate structure could not be controlled. Previously, in producing amorphous Ca-silicate by sol-gel [19] we found that the reaction of Ca with atmospheric $\mathrm{CO}_{2}$ to form phase separated calcium carbonate could be suppressed by drying the gel under vacuum. In addressing the Fe-Mg silicate ANP synthesis problem we have adapted this procedure to allow the controlled synthesis of Fe-Mg pyroxene silicate ANPs with low size dispersion and tuneable Fe content. In this paper we describe the necessary sol-gel procedure and present the results of laboratory characterisations using electron microscopy, synchrotron radiation and IR spectroscopy.

\section{Experimental}

\subsection{Sol-gel synthesis}

Silicate ANPs were produced in the beamline I11 support laboratory at the Diamond Light Source (UK). The sol-gel is formed by rapidly mixing two 0.1 molar solutions (18 M .cm deionised water) of (i) magnesium chloride $\left(\mathrm{MgCl}_{2}\right)$ and ferrous ammonium sulphate $\left(\mathrm{Fe}(\mathrm{II})\left(\mathrm{NH}_{4}\right)_{2}\left(\mathrm{SO}_{4}\right)_{2}\right)$ and (ii) sodium metasilicate $\left(\mathrm{Na}_{2} \mathrm{SiO}_{3}\right)$; prepared in stoichiometric ratios necessary to produce $\mathrm{Fe}_{x} \mathrm{Mg}_{1-x} \mathrm{SiO}_{3}$ target compositions with $x=0.1,0.2,0.3,0.4$, 0.5, 0.8 and 1.0. All reagents were analytical grade and used as supplied.

Upon mixing, blue-green gelatinous suspensions were formed which were magnetically stirred for 5 minutes to ensure complete mixing/reaction. If left to settle at room temperature, the gels gradually turn brown, resulting in a product with high Fe-oxide content. Thus, immediately after stirring, the suspensions were centrifuged and washed several times in deionised water to remove dissolved reactants. The gels were then transferred to covered 
alumina boats and dried under vacuum at $50{ }^{\circ} \mathrm{C}$ in a Carbolyte vacuum furnace purged with $\mathrm{N}_{2}$ gas prior to loading. Before the gels could be heated, the sample chamber was pumped overnight to $<10^{-6}$ mbar. During heating the pressure inside the vessel increased but by the end of the heating stage ( $\sim 6$ hours) had returned to below $10^{-6}$ mbar. The chamber was brought to a positive over pressure using $\mathrm{N}_{2}$ gas and the dry loose powder samples removed and stored under Ar in sealed vials inside a desiccator cabinet. From two 250 $\mathrm{ml}$ starting solutions a yield of approximately $2 \mathrm{~g}$ of powder was obtained. The powder colours ranged from very light brown for $x=0.1$ to dark brown for $x=1$.

Since vacuum drying was at relatively low temperature, the silicate ANPs are likely to be hydrated, either with bonded $\mathrm{OH}$ or trapped interstitial $\mathrm{H}_{2} \mathrm{O}$. Portions of the dried ANPs were therefore also annealed at 200,300, 400, 450, 500 and $650{ }^{\circ} \mathrm{C}$ under vacuum to determine the possible steps required to produce anhydrous product. The furnace chamber was evacuated to $\sim 2 \times 10^{-6}$ mbar and the ramp rate set to $10{ }^{\circ} \mathrm{C} \mathrm{min}^{-1}$. The set point temperatures were maintained for 12 hours, after which the furnace was switched off and left to cool to room temperature. The annealed powders were again stored under Ar.

\subsection{Characterisation measurements}

FTIR Transmission spectra $\left(6000-210 \mathrm{~cm}^{-1}\right)$ were measured for pressed pellets (0.5-1 mg of sample with $300 \mathrm{mg} \mathrm{KBr}$ pressed at $10 \mathrm{~T}$ for $4 \mathrm{~min}-$ utes) using the ESPOIRS instrument at IRAP in Toulouse. This comprises a Bruker VERTEX 70V spectrometer equipped with globar lamp, CsI beamsplitter and DLaTGS detector with CsI window. 
TEM analysis was performed using a FEI Tecnai G2-20 twin at the University of Lille electron microscopy centre. Small amounts of each composition were crushed in alcohol to form a suspension and a drop deposited on a carbon film supported by a copper TEM grid. Particle sizes were measured by bright field conventional nanoscale imaging, while X-ray energy dispersive spectroscopy (EDS) provided compositional analysis.

Synchrotron X-ray powder diffraction (SXPD) measurements were made on beamline I11 [20] at the Diamond Light Source (UK). Diffraction data out to $150^{\circ} 2 \theta$ were collected in $0.001^{\circ}$ intervals using $15 \mathrm{keV} \mathrm{X}$-rays and the diffractometer's multi-analyser crystal system in Debye-Scherrer geometry. Total X-ray scattering (TXS) was measured on beamline I15 at Diamond for samples loaded in $1 \mathrm{~mm}$ diameter Kapton tubes using $75.99 \mathrm{keV}$ X-rays focused down to $70 \mu \mathrm{m}$ and a Perkin Elmer 1621 EN area detector located $200 \mathrm{~mm}$ from the sample position. Data collection involved summing 200 frames, each comprising ten $1 \mathrm{~s}$ exposures. TXS data was transformed to radial pair distribution functions (PDFs) using the PDFgetX2 software ${ }^{2}$.

Fe K-edge X-ray absorption spectra were recorded in transmission on Diamond's B18 beamline [21, 22]. A few mg of each composition was mixed with $30 \mathrm{mg}$ of cellulose powder, lightly ground and pressed (10 T) into pellets $\sim 0.5 \mathrm{~mm}$ thick. Spectra were recorded between 6.912 and $7.961 \mathrm{keV}$ in 0.3 to $0.4 \mathrm{eV}$ intervals. Fe K-edge Spectra for library samples of hematite $\left(\mathrm{Fe}_{2} \mathrm{O}_{3}\right)$, magnetite $\left(\mathrm{Fe}_{3} \mathrm{O}_{4}\right)$ and pyrite $\left(\mathrm{FeS}_{2}\right)$ available at the beamline were also recorded.

\footnotetext{
${ }^{2}$ http://www.totalscattering.lanl.gov
} 


\section{Results}

Fig. 1a shows mid-IR spectra for the as dried powders. All the spectra contain three main contributions: the characteristic silicate O-Si-O bend and Si-O stretch bands $\left(\sim 250-800 \mathrm{~cm}^{-1}\right.$ and $\sim 800-1300 \mathrm{~cm}^{-1}$ respectively), plus a broad hydration band $\left(\sim 3500 \mathrm{~cm}^{-1}\right)$ with associated minor bands $\left(1300-1800 \mathrm{~cm}^{-1}\right.$ and $\left.\sim 5150 \mathrm{~cm}^{-1}\right)$. Although some variation in band widths can be seen, the two silicate bands are devoid of fine structure features, consistent with an absence of crystalline structure.

TEM analysis for selected compositions $(x=0.1,0.5$ and 0.8$)$ revealed amorphous nanoparticles with individual sizes ranging from 12 to $33 \mathrm{~nm}$ with a distribution peak at $\sim 20 \mathrm{~nm}$ (e.g. Fig. 2) that was found to be independent of composition. EDS for large and small sampling areas $(>0.5 \times 0.5$

$\mu \mathrm{m}^{2}$ and $100 \times 100 \mathrm{~nm}^{2}$ ) showed good chemical homogeneity with achieved compositions lying close to the target formula (e.g. Fig. 3). The spread in composition was found to be largest for low Fe concentration, suggesting Fe incorporation may be higher in some particles and lower in others. Trace level quantities of $\mathrm{Na}, \mathrm{Cl}, \mathrm{K}$ and $\mathrm{Ca}$ left over from the sol-gel were detected; however on a scale up to $10^{-3} \mu \mathrm{m}^{3}$ the as dried ANPs were found to be homogeneous with no phase separation to $\mathrm{SiO}_{2}$ or $\mathrm{Mg} / \mathrm{Fe}-$ oxide.

SXPD (e.g. Fig. 4) showed only the broad features characteristic of a material that at the atomic level is either amorphous or nanostructured. Only minor variations exist between compositions, indicating a relatively consistent particle structure. This is shown in more detail in Fig. 5a which shows PDFs obtained from the TXS measurements. All the PDFs show a very similar structure in terms of feature number, position and profile, although there 
is a clear change in the relative strengths of the two peaks at $\sim 1.6$ and $\sim 2.0$ $\AA$ as the Fe content increases. For the $x=0.8$ PDF the structural ordering appears to extend over a slightly longer range which may reflect some minor difference in synthesis conditions. Even so, at short-range the $x=0.8 \mathrm{PDF}$ is still very similar to the other compositions. The Fe K-edge absorption spectra (Fig. 6a) for the as dried ANPs are also all very similar, displaying a pre-edge peak $(\mathrm{A})$ due to $1 s \rightarrow 3 p$ transitions, a weak shoulder at the bottom of the absorption rise due to ligand-to-metal charge transfer (LMCT, [26]) and a sharp absorption rise to a large narrow peak (B) followed by a weaker secondary peak $(\mathrm{C})$, both of which are due to multiple scattering (MS). In transition metal oxides, these post-edge features are due to hybridization of the central atom's $4 p$ orbitals with the $3 d$ octahedral molecular orbitals of the neighbouring $\mathrm{O}$ atoms and MS calculations for silicates [27, 28, 29] show a minimal cluster of a central Fe with six nearest $\mathrm{O}$ neighbours is sufficient to account for both the main peak (B) and the broad peak (C); while including larger cluster sizes involving higher shells allows for the appearance of the pre-peak (A) which is therefore an indicator of medium-range structure.

\section{Discussion}

\subsection{Structural environment}

The characterisation measurements above confirm the production method

yields a set of homogeneous silicate ANPs with pyroxene compositions and atomic-level structures that are very closely related. A semi-quantitative measure of the similarities between the ANP structures can be obtained from the PDF data [25] using the Pearson product-moment correlation, $R$, 
calculated pair-wise for all PDF datasets,

$$
R=\frac{1}{1-n} \sum_{i=0}^{n}\left(\frac{X_{i}-\bar{X}}{\sigma_{x}}\right)\left(\frac{Y_{i}-\bar{Y}}{\sigma_{y}}\right)
$$

where $\bar{X}$ and $\bar{Y}$ are the mean values of datasets $X$ and $Y$ and $\sigma_{x}$ and $\sigma_{y}$ their standard deviations. $R$ is insensitive to differences in amplitude scale and for each pair returns a value between 1 (fully correlated) and -1 (fully anticorrelated) with 0 representing uncorrelated. Thus $R$ allows us to gauge how similar/dissimilar the structures are without knowing specific details of atomic coordinates and bond angles etc. [25].

The results of comparing the structures for the as dried ANPs are given in Table 1 (top), calculated using the CORRELATE function in IDL. For compositions up to $x=0.5$ the structures are highly correlated irrespective of composition. Although the $x=0.8 \mathrm{PDF}$ shows structural order extending over a longer range, its correlation with the $x<0.5$ compositions is still reasonably high and its correlation with $x=1.0$ very high, suggesting a structure that is intermediate of $x=0.5$ and 1.0. In general, the correlation is high across all the compositions, suggesting that any variation in structure due to increased Fe content is both gradual and proportionate.

The coherent scattering domain size (CSD) is the maximum distance over which structural correlations can be discerned and is represented by the distance at which the amplitude of the PDF oscillations diminish to zero. From Fig. 5a the CSD for the as dried ANPs is $\sim 20 \AA$ for all $x(\sim 30$ $\AA$ for $x=0.8$ ), such that the silicate ANPs are only ordered on a scale that is $\sim 10 \times$ smaller than their average physical dimension determined by TEM. The CSD reduced further by a factor of $\sim 2$ when the ANPs were 
annealed in vacuum at temperatures in the range of $\sim 450{ }^{\circ} \mathrm{C}$ to $\sim 500{ }^{\circ} \mathrm{C}$, with temperatures above $500{ }^{\circ} \mathrm{C}$ causing the CSD length to increase as the ANPs start to crystallise (Fig. 5b). The reduction in the CSD also brought the $x=0.8$ structure into line with the other compositions (Fig. 5c), with all the structures appearing well correlated with each other (Table 1 bottom). Independent of Fe content, annealing the ANPs below their crystallisation temperature results in amorphous particles that are structurally very similar.

The first two peaks in the PDFs in Fig. 5 at $\sim 1.6 \AA$ and $\sim 2.0 \AA$ correspond to the $\mathrm{Si}-\mathrm{O}$ and $\mathrm{M}-\mathrm{O}$ distances $(\mathrm{M}=\mathrm{Mg}$ or $\mathrm{Fe})$. The $\mathrm{Si}-\mathrm{O}$ bondlength (Fig. 7a), obtained from the first derivative of the PDF, exhibits no linear compositional dependency as it is also susceptible to other physical factors such as strain and tetrahedral polymerisation [23]. The M-O bond similarly obtained however shows a clearer dependency (Fig. 7b), generally shortening and tending towards a constant value as $x$ increases. The PDF derived M-O distances in Fig. 7b, though indicative of octahedral bondlengths, are of course averaged over both $\mathrm{Mg}-\mathrm{O}$ and Fe-O. Values for the Fe-O distances (Fig. 7c) obtained from first shell fits to the K-edge EXAFS signal using the Fe-O rocksalt structure show a much smaller range of variation (1.95 to $2.00 \AA)$ and are closer to octahedral coordination $[40,41]$ than the $1.80 \AA$ typical of tetrahedral bonding, suggesting that few, if any, of the Fe atoms are network formers. Other instances of $\mathrm{Fe}^{2+}$ and $\mathrm{Fe}^{3+}$ occupying similar octahedral sites in non-crystalline Fe-silicate environments (e.g. glasses) have also been reported [24].

The absorption edge (B) feature for the as dried ANPs (Fig 6a) appears singular, with its crest at $\sim 7133 \mathrm{eV}$. However in $\alpha$-hematite $\left(\mathrm{Fe}_{2} \mathrm{O}_{3}\right)$ this 
feature consists of a main peak and a weaker lower energy shoulder. The Fe in $\alpha$-hematite are distributed in two parallel sets of alternating layers of equivalent octahedral sites which show different degrees of distortion [30, 31]. This manifests as a weak shoulder corresponding to the interaction of Fe with O atoms at $2.115 \AA$, while the main peak is due to interaction with $\mathrm{O}$ atoms at $1.945 \AA[31,32]$. The energies of these two features are typically $\sim 7131$ $\mathrm{eV}$ and $\sim 7134 \mathrm{eV}$. For the as dried ANPs, the main peak lies close to $\sim 7134$ $\mathrm{eV}$ and, apart from the $x \geq 0.5$ compositions, there is no significant lower energy shoulder. For the high $x$ ANPs the shoulders are very weak and therefore also indicate that for the as dried ANPs the Fe atoms sit mostly within regular octahedral sites. However, for the $450{ }^{\circ} \mathrm{C}$ annealed ANPs (Fig. 6b) the shoulder is much stronger, with (B) feature maxima close to 7132 and $7134 \mathrm{eV}$, showing the formation of two distinct octahedral environments. Thus, while annealing reduces long-range ordering by reducing the CSD size, it does result in an increase in the short-range ordering.

\subsubsection{Fe oxidation state}

As well as being an indicator for medium-range structure, the pre-peak (A) in the absorption spectrum (Fig. 6) also carries information regarding the oxidation state of the incorporated Fe, with a $1.4 \pm 0.1 \mathrm{eV}$ difference between its centroid position being observed in $\mathrm{Fe}^{2+}$ and $\mathrm{Fe}^{3+}$ minerals $[33,34,35]$. Since hematite, magnetite and pyrite are known to have $100 \%, 66.7 \%$ and 0 $\% \mathrm{Fe}^{3+}$, respectively, their pre-peak positions were taken from the measured library spectra and used to construct a calibration line from which $\mathrm{Fe}^{3+} / \Sigma \mathrm{Fe}$ was calculated for each nanosilicate. This method has been applied to a wide range of minerals and glasses [35, 36, 37], although it can potentially be 
subject to error if the difference in site geometry between the reference standards and the unknowns is too large $[35,36]$. The centroid positions however do allow systematic trends to be identified and Fig. 8 shows $\mathrm{Fe}^{3+} / \Sigma \mathrm{Fe}$ for the as dried and annealed ANPs as a function of composition. For $x \gtrsim 0.5$, $\mathrm{Fe}^{3+} / \Sigma \mathrm{Fe}$ appears much the same in both, while annealing at $450{ }^{\circ} \mathrm{C}$ significantly reduces the level of $\mathrm{Fe}^{3+}$ for $x \lesssim 0.5$. The variation in $\mathrm{Fe}^{3+} / \Sigma \mathrm{Fe}$ for the lower $x$ compositions may be related to the possible variation of Fe incorporation between particles inferred by the EDS analysis. Recent studies of aqueously altered amorphous Fe-Mg silicate $\left(\mathrm{Fe}_{1.1} \mathrm{Mg}_{0.9} \mathrm{SiO}_{4.15} \mathrm{H}_{0.3}\right)$, initially produced by pulsed laser deposition and containing only $\mathrm{Fe}^{2+}$, found $\mathrm{Fe}^{3+} / \Sigma \mathrm{Fe}$ values of $\sim 60-75 \%[38,39]$ suggesting the average $\mathrm{Fe}^{3+} / \Sigma \mathrm{Fe} \sim 50$ $\%$ for the ANPs here originates in the hydrolysis reactions that occur during the initial gel formation.

\subsection{Effect of Fe on IR spectrum}

For the as dried ANPs, the Si-O stretch band (Fig 1a) exhibits a broadening with increasing $x$, while its peak position $\left(1022 \mathrm{~cm}^{-1}\right)$ varies only by $\sim 2 \mathrm{~cm}^{-1}$. However, for the ANPs annealed at $450^{\circ} \mathrm{C}$ (Fig. 1b) it is the band width that shows little variation while the peak shifts from 1023 to $1046 \mathrm{~cm}^{-1}$ for $x \geq 0.8$. This is also accompanied by a shift in the bending mode peak to lower wavenumbers as a new peak appears in the form of a shoulder at $458 \mathrm{~cm}^{-1}$. The presence in the as dried ANP's bending mode of two peaks points to the silicate network not being fully built, with a lower degree of tetrahedral polymerization. However, this needs to be considered in conjunction with the PDF data. In bulk amorphous silicates there are two basic models: the Zachariasen-Warren model [42]-[45], in which Si-O 
tetrahedra are linked together in a statistically disordered way to form a large-scale continuous random network (CRN) with no long-range periodicity; and the "microcrystal" model attributed to Lebiediev and co-workers [46]-[49], in which the CRN links between the surfaces of ultrafine $(\sim 15-20$ $\AA$ ) crystalline domains [50]. Since the PDF data shows ordering on the 10-20 $\AA$ scale, the double peaked bending mode may derive from a structure where the nano sized crystalline domains and interlinking CRN have differing polymerisation. Since the domain structure below $10 \AA$ does not change with annealing, further network building by polymerisation presumably only occurs within the CRN surrounding the nano domain (since this is observed to change when the CSD shrinks during annealing), presumably via the linking together of tetrahedra made possible by the loss of water and/or bonded $\mathrm{OH}$ $[23,51]$ and also resulting in a change in the Si-O peak position. However, although our investigations support the domain-structured model, neither the X-ray nor the IR data allow us to distinguish between ANPs with a single core-shell structure or, given the small size of the ordered regions relative to the physical particle size, ANPs containing multiple nano domains linked via a CRN.

\subsection{ANP Hydration}

In Fig. 1a the main hydration band is the strong, broad asymmetric feature peaking at $\sim 3500 \mathrm{~cm}^{-1}$. The high $x$ ANPs exhibit a broader asymmetry on the low wavenumber side with a strong side feature at $\sim 2300 \mathrm{~cm}^{-1}$; while the lower $x$ ANPs show a high wavenumber tail at $\sim 3850 \mathrm{~cm}^{-1}$. The band itself is attributable to interstitial $\mathrm{H}_{2} \mathrm{O}$ and ( $\mathrm{Si}$ ) O-H groups in differing structural environments [52] and clearly varies with Fe content. The sharp band 
located at $\sim 1650 \mathrm{~cm}^{-1}$ is due to the $\mathrm{H}-\mathrm{O}-\mathrm{H}$ bending mode of coordinated and/or adsorbed water [53], while the band at $\sim 5150 \mathrm{~cm}^{-1}$ is a combination band of molecular water $[54,55]$. Annealing at $450{ }^{\circ} \mathrm{C}$ reduces the $3500 \mathrm{~cm}^{-1}$ band strength by $\sim 30 \%$ with similar behaviour for the 1650 and $5150 \mathrm{~cm}^{-1}$ bands (Fig. 1b). However, doubling the annealing time produced no further change in the shape, position or strength of any of the hydration bands. Annealing at higher temperatures could potentially remove more, or all, $\mathrm{H}_{2} \mathrm{O}$ and bonded $\mathrm{OH}$ but as Fig. 5b suggests it will, depending on temperature, also result in increased levels of crystallisation. Some level of persistent hydration is therefore likely to be an inherent feature of sol-gel produced ANPs, but could potentially play an important role in defining their functional or reactive behaviour in future applications.

\section{Conclusions}

The tendency for Fe to form oxide phases means that its incorporation during materials syntheses can be problematic and the primary objective of the work reported here was to develop a means of reliably synthesising amorphous pyroxene nanosilicates with controlled $\mathrm{Fe} / \mathrm{Mg}$ content. In doing so we have developed a robust, well controlled and easy to execute method of producing homogeneous, monodispersed, Fe-bearing nanosilicates in which the Fe atoms are fully incorporated into the amorphous silicate structure. To verify this we have characterised the structural and spectroscopic prop-

erties of the nanosilicates showing that our relatively facile sol-gel based process produces amorphous nanoparticles with selectable Fe content across the $\mathrm{Fe}_{x} \mathrm{Mg}_{1-x} \mathrm{SiO}_{3}$ range. The nanoparticles contain ordered silicate nan- 
odomains typically $20 \AA$ in size which are reduced further by annealing the particles in vacuum below their crystallisation temperature. Although this reduces correlations in their long-range structure, annealing does increase the ordering of the local short-range environment. The use of a sol-gel as a precursor means that the particles retain a degree of hydration. However, by controlling the annealing temperature it should be possible to selectively produce particles with differing degrees of crystallinity, depending on the requirements of any potential application. Although we have not yet conducted an exhaustive investigation of the optimum necessary conditions required for the transformation of a gel into solid nanoparticles, we believe it is in the application of high vacuum during the drying step that the particle size is determined as this likely interferes with, or disrupts, the post-gelation monomer aggregation process. Tests using a vacuum oven (Thermo Scientific) to dry the gels under medium vacuum (0.01 mbar compared to $10^{-6}$ mbar used in this work) resulted in the formation of large irregular solids several $\mathrm{mm}$ in diameter, similar to the material produced from gels dried in air [56]. Thus, by varying either the pumping rate, or the base vacuum pressure, it may prove possible to vary the size distribution. Alternatively, although the present work is aimed at producing Fe-Mg pyroxene silicate ANPs with little, or no, contaminating species, if compositional purity is not a major consideration size control might potentially be achieved by the inclusion of additional steps and/or additives in the production process. For example $\mathrm{SiO}_{2}, \mathrm{TiO}_{2}$ and $\mathrm{Zn}_{2} \mathrm{SiO}_{4}$ nanoparticles with uniform sizes have been produced via sonochemical methods utilising ultrasonic treatment and Schiff base additives 
[57]- [60], while "green" $\mathrm{Fe}_{2} \mathrm{O}_{3} / \mathrm{CuFe}_{2} \mathrm{O}_{4}$ ferromagnetic nanocomposite oxides have been produced via sol-gel-autocombustion in which an onion-derived reductant additive was used [61].

\section{Acknowledgements}

This work was supported by Diamond Light Source (beamtime allocations EE8692 and SP8614) and by the ANR: project CIMMES (ANR-11-BS56029-01). SJD acknowledges financial support by Diamond Light Source and Keele University. HL and CD thank the Chevreul Institute, the European FEDER and the Région Nord-Pas-de-Calais (France).

\section{References}

[1] X. Changsheng, H. Junhui, W. Run, X. Hui. NanoStructured Mater. 11 (1999) 1061.

[2] V.V. Hoang, D. Ganguli. Physics Reports 518 (2012) 81.

[3] Machala, L., Zboril, R., Gedanken, A. J. Phys. Chem. B 111 (2007) 4003.

[4] M. Rapp, I.Strelnikova, J. Gumbel, Adv. Space Res. 40 (2007) 809.

[5] M.E. Hervig, L.L. Gordley, L.E. Deaver, et al. Geophys. Res. Lett. 36 (2009) L18805.

[6] J.M.C. Plane, Chem. Rev. 103 (2003) 4963.

[7] R. W. Saunders, S. Dhomse, W. S. Tian, et al. Atmos. Chem. Phys. 12 (2012) 4387. 
[8] K. Murata, T. Takakura, H. Chihara, et al. Astrophys. J. 696 (2009) 1612.

[9] A. Kuzmin, J. Chaboy. IUCrJ 1 (2015) 571.

[10] J. Dorschner, Th. Henning, Astrophys, Space Sci. 128 (1986) 47.

[11] W. Krätschmer In Experiments on Cosmic Dust Analogues. Bussoletti, E., Fusco, C. Longo, G. (Eds.) Kluwer, Dordrecht, p. 95

[12] C. Jäger, H. Mutschke, B. Begemann, J. Dorschner, Th. Henning, Astron. Astrophys. 292 (1994) 641.

[13] J. Dorschner, B. Begemann, Th. Henning, et al. Astron. Astrophys. 300 (1995) 503.

[14] J. Matsuno, A. Tsuchiyama, a. Miyake, et al., Astrophys. J. 792 (2014) 136.

[15] F.J.M. Rietmeijer, J.A. Nuth, J.M. Karner, Astrophys. J. 527 (1999) 395.

[16] J.R. Brucato, V. Mennella, L. Colangeli, et al., Planet. Space Sci. 50 (2002) 829 .

[17] E. Anders, N. Grevesse, Geochim. Cosmochim. Acta 53 (1989) 197.

[18] K. Murata, H. Chihara, A. Tsuchiyama, et al., Astrophys. J. 668 (2007) 285.

[19] S.P. Thompson, S.J. Day, J.E. Parker, A. Evans, C.C. Tang J. Non-cryst. Sol. 358 (2012), 885. 
[20] S.P. Thompson, J.E. Parker, J. Potter, et al., Rev. Sci. Instrum. 80 (2009) 075107.

[21] A.J. Dent, G. Cibin, S. Ramos, et al., J. Phys. Conf. Ser. 190 (2009) 012039 .

[22] A.J. Dent, G. Cibin, S. Ramos, et al., J. Phys. Conf. Ser. 430 (2013) 012023.

[23] S.P. Thompson, J.E. Parker, C.C. Tang. Astron. Astrophys. 545 (2012) A60.

[24] F. Farges, Y. Lfrère, S. Rossano, et al. J. Non-Cryst. Sol. 244 (2004) 176.

[25] T. Davis, M. Johnson, S.J.L. Billinge, Cryst. Growth Des. 13 (2013) 4239.

[26] O. Haas, U.F. Vogt, C. Soltmann, et al. Mater. Res. Bull. 44 (2009) 1397.

[27] Z.Y. Wu, D.C. Xian, T.D. Hu, et al, Phys. Rev.B 70 (2004) 033104.

[28] Z.Y. Wu, G. Ouvrard, S. Lemaux, et al., Phys. Rev. Lett. 77 (1996) 2101.

[29] Z.Y. Wu, G. Ouvrard, P. Moreau, C.R. Natoli, Phys. Rev. B 55 (1997) 9508.

[30] D.H. Lindsley, Rev. Mineral. 3 (1976) L1. 
[31] R.L. Blake, R.E. Messecick, T. Zoltai, L.W. Finger, Am. Miner. 51 (1996) 123.

[32] E. Paris, A. Mottana, P. Mattias, Min. Petrol. 45 (1991) 105.

[33] G.A. Waychunas, M.J. Brown, M.J. Apted, Phys. Chem. Minerals 10 (1983) 1 .

[34] S. Bajt, S.R. Sutton, J.S. Delaney, Geochim. Cosmochim. Acta 58 (1994) 5209 .

[35] P.-E. Petit, F. Farges, M. Wilke, V.A. Solé, J. Synchrotron Rad. 8 (2001) 952.

[36] M.D. Dyar, M.E. Gunter, J.S. Delaney, et al. Canadian Miner. 40 (2002) 1375.

[37] J.S. Delaney, S.R. Sutton, S. Bajt, Geol. 26 (1998) 139.

[38] C. Le Guillou, H.G. Changela, R. Dohmen, et al. Proc. Lunar Planet. Sci., 45th Conf. (2014) 2052.

[39] C. Le Guillou, R. Dohmen, D. Rogalla, et al. Chemical Geology 412 (2015) 179.

[40] S. Ghose, V. Schomaker, R.K. McMullan, Z. Krist. 176 (1986) 159.

[41] D. Li, M. Peng, T. Murata, Can. Mineral. 37 (1999) 199.

[42] W.H. Zachariasen, J. Am. Ceram. Soc. 17 (1932) 3841.

[43] B.E. Warren, Z. Krist. 86 (1993) 349. 
[44] B.E. Warren B. E., 1934, J. Am. Ceram. Soc. 17 (1934) 249.

[45] B.E. Warren, H. Krutter, O. Morningstar J. Am. Ceram. Soc. 19 (1936) 202.

[46] A.A. Lebiediev, Trans. Opt. Inst. Petr. 2 (1921) 10.

[47] J.T. Randall, H.P. Rooksby, B.S. Cooper, J. Soc. Glass Technol. 14 (1930) 219 .

[48] J.T. Randall, H.P. Rooksby, B.S. Cooper, Z. Krist. 75 (1930) 196.

[49] N. Valenkow, E. Porai-Koshitz, Z. Krist. 95 (1936) 195.

[50] H. Verweij, W.L. Konijnendijk, J. Am. Ceramic Soc. 59 (1976) 517.

[51] S.P. Thompson, J.E. Parker, S.J. Day, et al. Mon. Not. Roy. Astron. Soc. 434 (2013) 2582.

[52] A.M. Efimov, V.G. Pogareva, A.V. Shashkin, J. Non-Cryst. Solids 332 (2003) 93.

[53] M.S. Augsberger, E. Strasser, E. Perino, et al., J. Phys. Chem. Solids 59 (1998) 175.

[54] M. Nowak, H. Behrens, Geochimica et Cosmochimica Acta 59 (1995) 3445.

[55] J. Madejová, M. Pentrák, H.Pálková, P. Komadel, Vib. Spectr. 49 (2009) 211. 
[56] S.P. Thompson, C. Verrienti, S. Fonti, V. Orofino, A. Blanco, Adv. Space Res. 39 (2007) 375.

[57] M. Masjedi-Arani, M. Salavati-Niasari, D. Ghanbari, G. Nabiyouni, Ceramics International 40 (2014) 495.

[58] M. Masjedi-Arani, D. Ghanbari, M. Salavati-Niasari, S. Bagheri, J. Cluster Sci. (2016) 27, 39.

[59] M. Masjedi, N. Mir, E. Noon, T. Gholami, M. Salavati-Nissari, Supperlattices and Microstrusctures (2013) 62, 30.

[60] M. Masjedi-Arani, M. Salavati-Niasari, Ultrasonics Sonochemistry (2016) 29226.

[61] F. Ansari, A. Sobhani, M. Salavati-Nissari, J. Magnetism and Magnetic Materials (2016) 410, 27. 
Table 1: Pearson product-momentum correlation, $R$, calculated out to $30 \AA$ from the $\underline{\mathrm{Fe}_{x} \mathrm{Mg}_{1-x} \mathrm{SiO}_{3} \text { PDFs. }}$

\begin{tabular}{|c|c|c|c|c|c|c|c|}
\hline \multicolumn{8}{|c|}{ As dried at $50{ }^{\circ} \mathrm{C}$} \\
\hline & $x=0.1$ & $x=0.2$ & $x=0.3$ & $x=0.4$ & $x=0.5$ & $x=0.8$ & $x=1.0$ \\
\hline$x=0.1$ & 1.000 & 0.979 & 0.972 & 0.917 & 0.943 & 0.677 & 0.845 \\
\hline$x=0.2$ & & 1.000 & 0.971 & 0.975 & 0.963 & 0.715 & 0.877 \\
\hline$x=0.3$ & & & 1.000 & 0.928 & 0.988 & 0.714 & 0.916 \\
\hline$x=0.4$ & & & & 1.000 & 0.948 & 0.744 & 0.892 \\
\hline$x=0.5$ & & & & & 1.000 & 0.766 & 0.959 \\
\hline$x=0.8$ & & & & & & 1.000 & 0.843 \\
\hline$x=1.0$ & & & & & & & 1.000 \\
\hline \multicolumn{8}{|c|}{ Annealed at $450{ }^{\circ} \mathrm{C}$} \\
\hline & $x=0.1$ & $x=0.2$ & $x=0.3$ & $x=0.4$ & $x=0.5$ & $x=0.8$ & $x=1.0$ \\
\hline$x=0.1$ & 1.000 & 0.995 & 0.978 & 0.973 & 0.940 & 0.863 & 0.801 \\
\hline$x=0.2$ & & 1.000 & 0.974 & 0.988 & 0.947 & 0.884 & 0.819 \\
\hline$x=0.3$ & & & 1.000 & 0.974 & 0.984 & 0.920 & 0.882 \\
\hline$x=0.4$ & & & & 1.000 & 0.972 & 0.937 & 0.883 \\
\hline$x=0.5$ & & & & & 1.000 & 0.972 & 0.948 \\
\hline$x=0.8$ & & & & & & 1.000 & 0.988 \\
\hline$x=1.0$ & & & & & & & 1.000 \\
\hline
\end{tabular}




\section{Figure Captions}

Fig. 1 FTIR spectra of amorphous Fe/Mg silicate ANPs in the 6000-210 $\mathrm{cm}^{-1}$ range (a) dried at $50{ }^{\circ} \mathrm{C}$; (b) annealed at $450{ }^{\circ} \mathrm{C}$. All spectra shown normalized to the peak maximum of the $\mathrm{Si}-\mathrm{O}$ stretching mode at $\sim 1000 \mathrm{~cm}^{-1}$.

Fig. 2 TEM image for $\mathrm{Fe}_{0.1} \mathrm{Mg}_{0.9} \mathrm{SiO}_{3}$ nanoparticles dried at $50{ }^{\circ} \mathrm{C}$. Size distribution and electron diffraction shown as insets.

Fig. 3 Fe-Mg-Si ternary diagrams showing measured compositions for (a) $\mathrm{Fe}_{0.1} \mathrm{Mg}_{0.9} \mathrm{SiO}_{3}$ and (b) $\mathrm{Fe}_{0.5} \mathrm{Mg}_{0.5} \mathrm{SiO}_{3}$ nanosilicates. The small difference in composition between the large and small sampling sizes is due to a partial volatilization of $\mathrm{Mg}$ during the electron beam analysis.

Fig. 4 Synchrotron X-ray powder diffraction patterns for selected amorphous $\mathrm{Fe}_{x} \mathrm{Mg}_{1-x} \mathrm{SiO}_{3}$ nanoparticles dried at $50{ }^{\circ} \mathrm{C}$.

Fig. 5 Pair distribution functions (a) for each as dried silicate composition; (b) for the $x=0.5$ composition as a function of annealing temperature and (c) for each composition after annealing at $450{ }^{\circ} \mathrm{C}$.

Fig. 6 Fe K-edge spectra for silicate ANPs for $x=0.1$ to 1.0 (offset in y-axis direction in order of increasing $x$ ) (a) dried at $50{ }^{\circ} \mathrm{C}$ and (b) annealed at $450{ }^{\circ} \mathrm{C}$, showing pre-edge feature (A), main absorption edge crest (B) and post edge structure (C) (see text for discussion).

Fig. 7 (a) Si-O distance $( \pm 0.005 \AA)$ as a function of Fe content for as dried ANPs (filled circles) and ANPs annealed at $450{ }^{\circ} \mathrm{C}$ (open circles) determined from PDF; (b) Variation of the $\mathrm{M}-\mathrm{O}$ ( $\mathrm{Fe} / \mathrm{Mg}$ metal to $\mathrm{O}$ ) 
distance $( \pm 0.005 \AA)$ determined from PDFs as a function of Fe content and annealing temperature; (c) Fe-O distances ( $\pm \sim 0.01 \AA$ ) determined from Fe K-edge EXAFS (note: plots rotated for review format only).

Fig. $8 \mathrm{Fe}^{3+} / \Sigma \mathrm{Fe}$ as a function of composition for ANPs dried at $50{ }^{\circ} \mathrm{C}$ (filled circles) and annealed at $450{ }^{\circ} \mathrm{C}$ (open circles). 

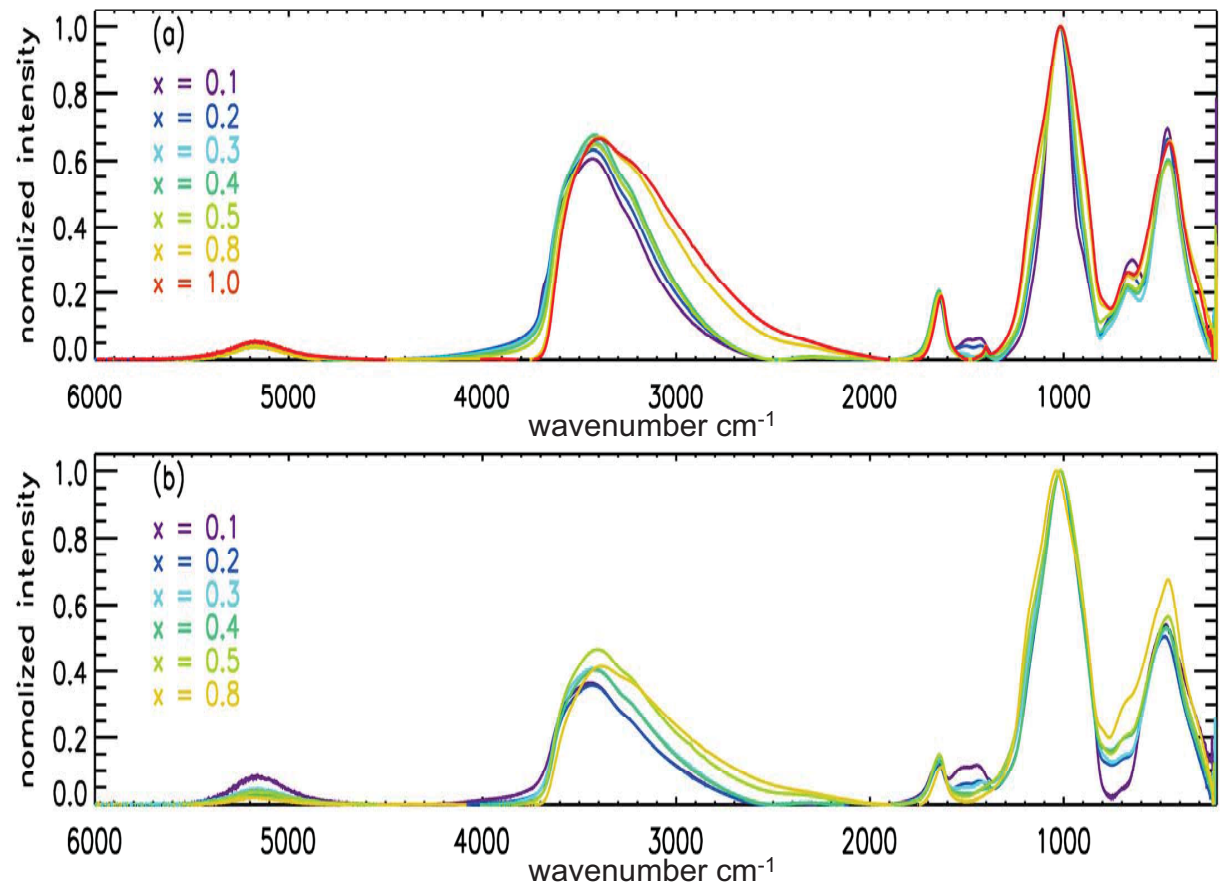

Figure 1: 


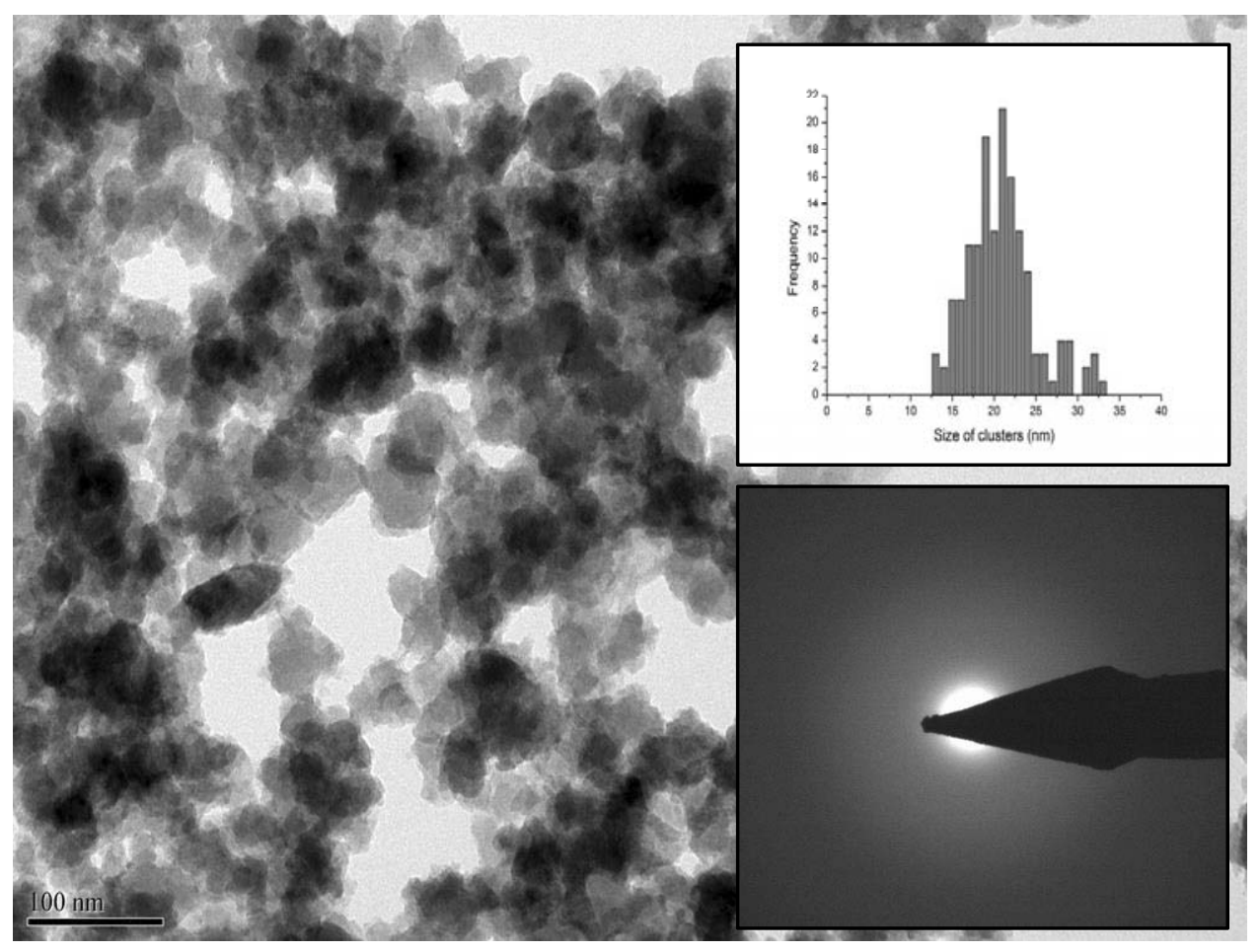

Figure 2: 

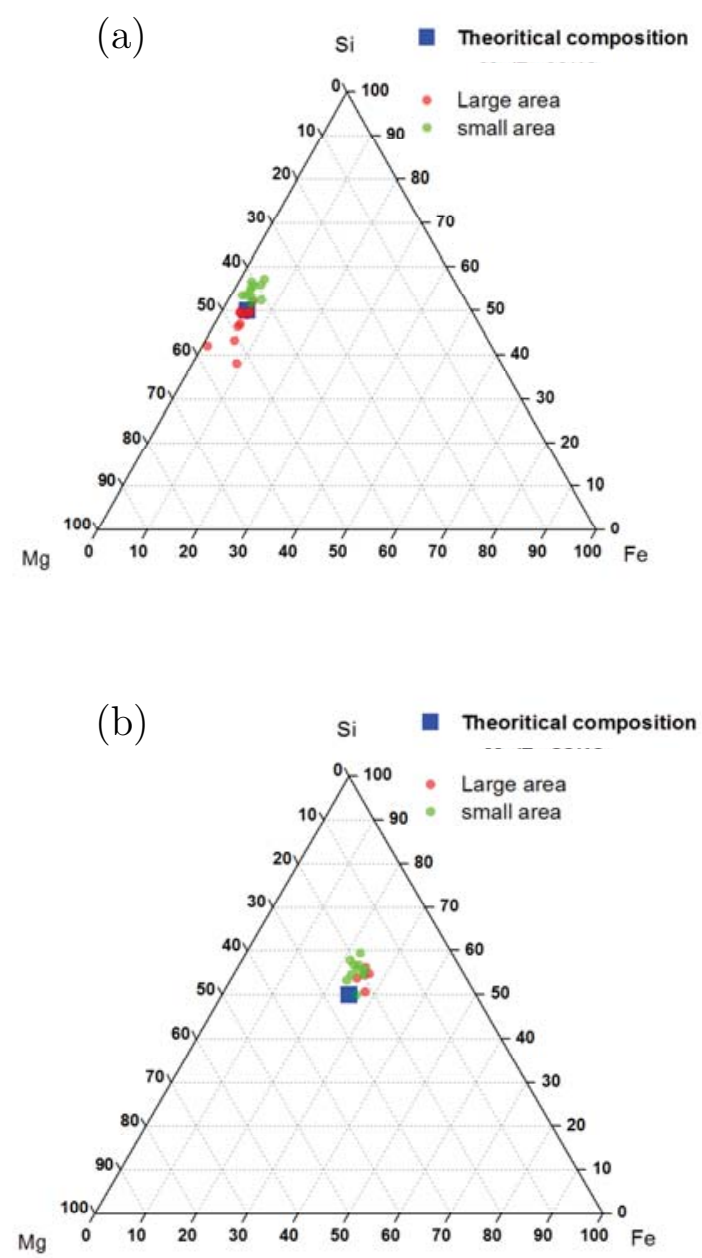

Figure 3: 


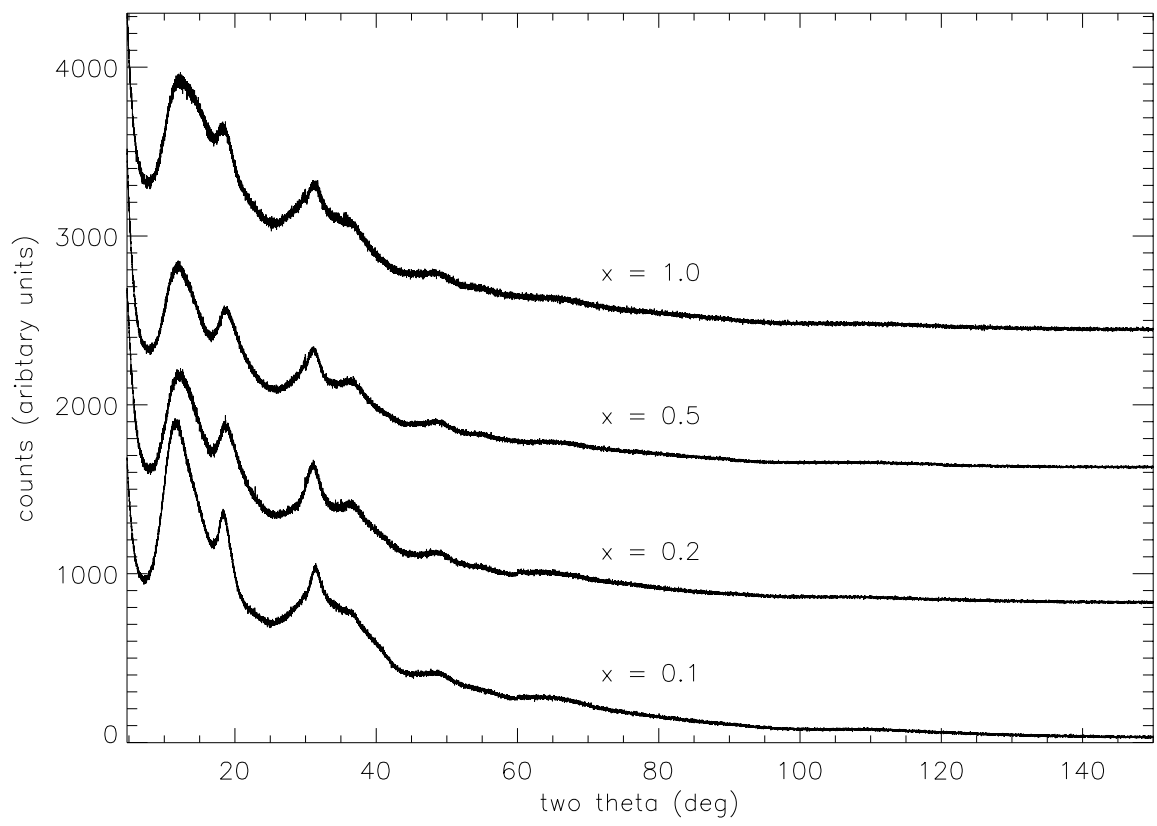

Figure 4: 
(a)

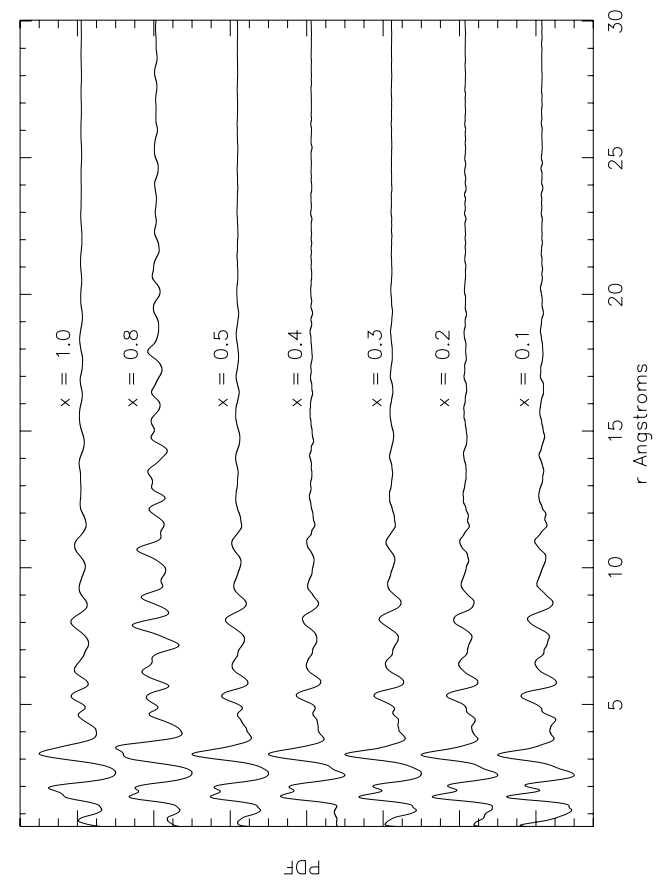

(c)

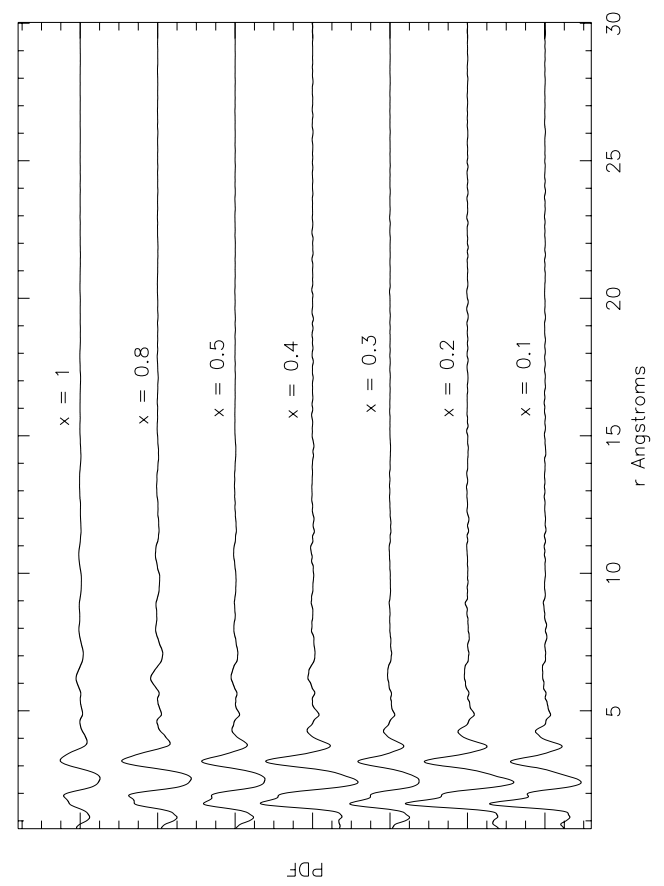

(b)

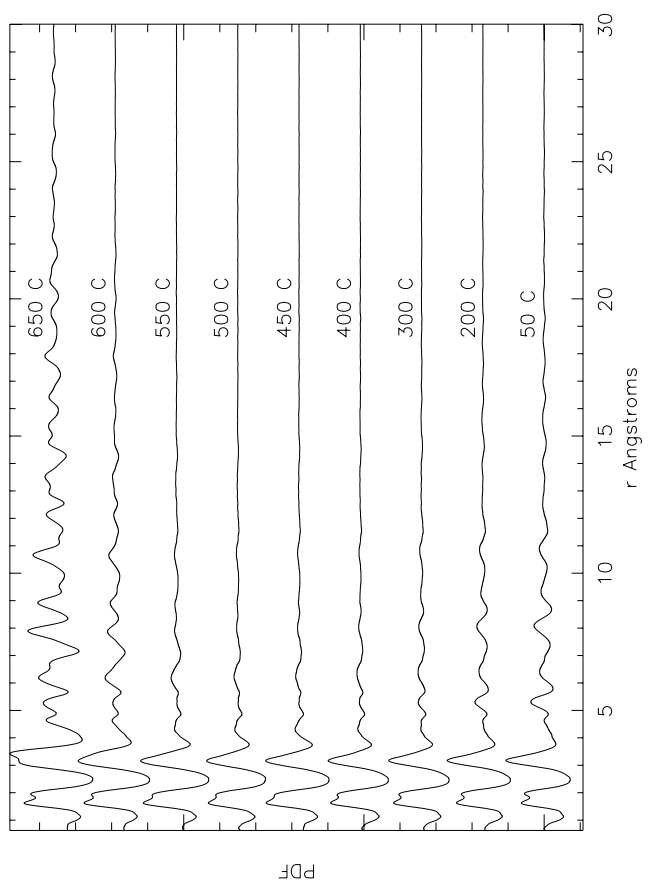

Figure 5: 
(a)

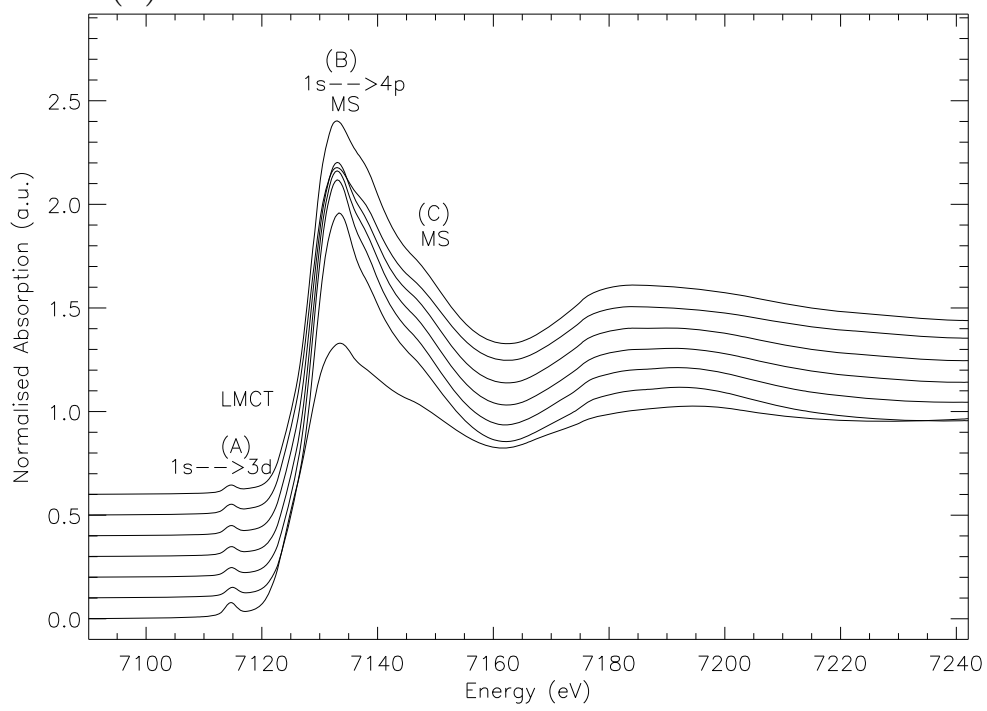

(b)

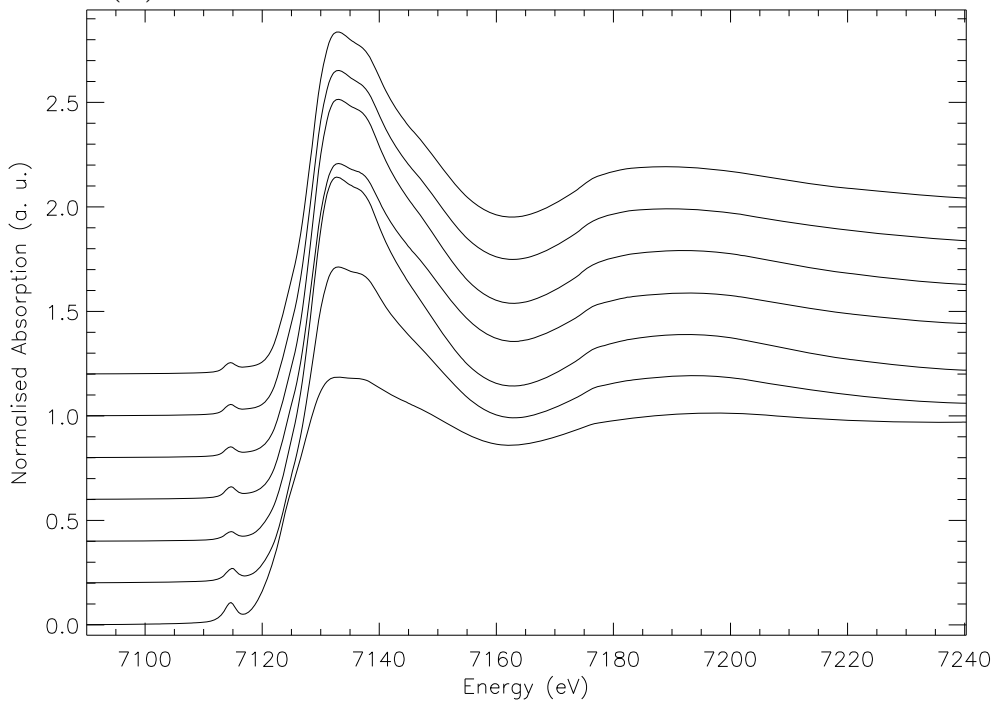

Figure 6: 

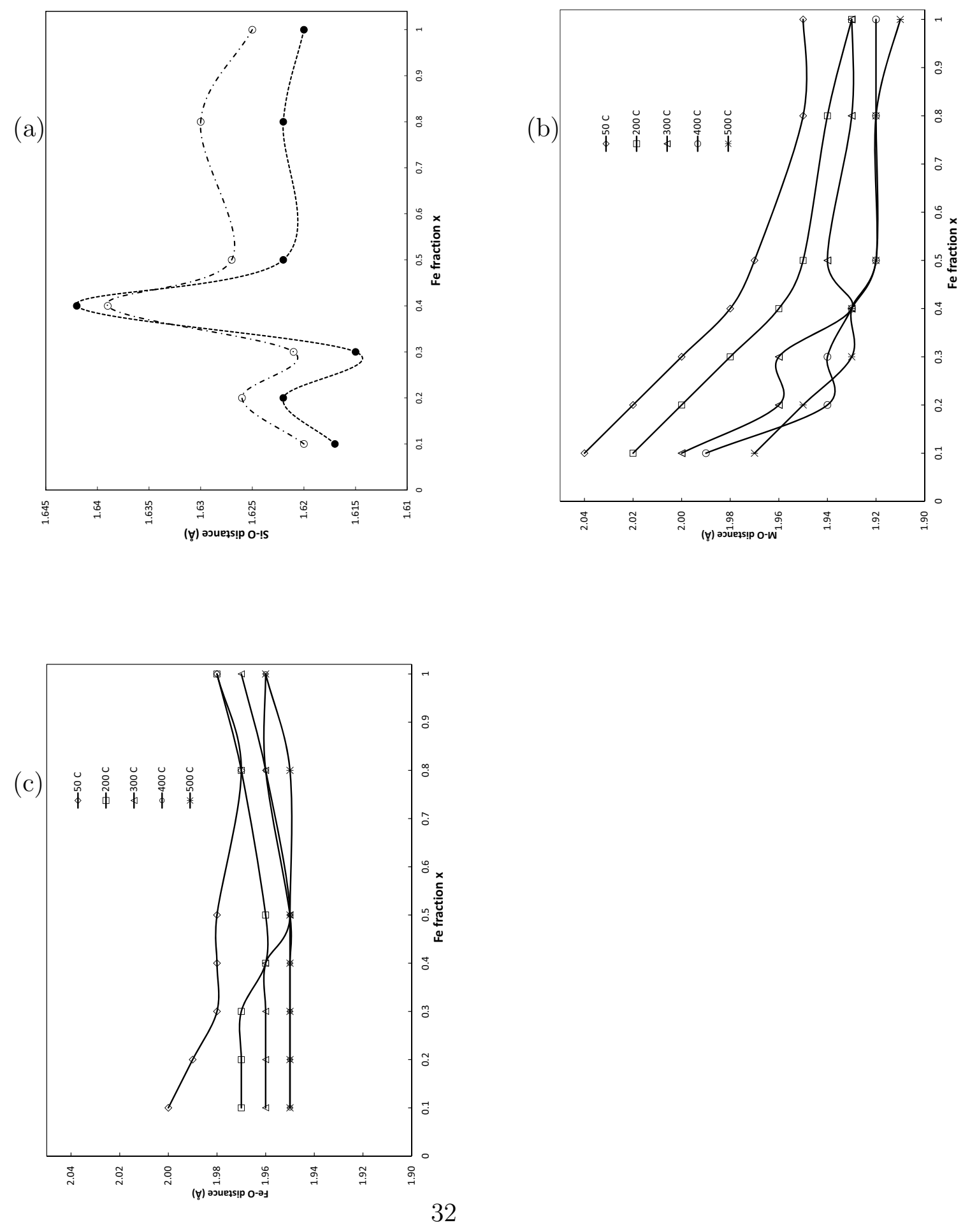

Figure 7: 


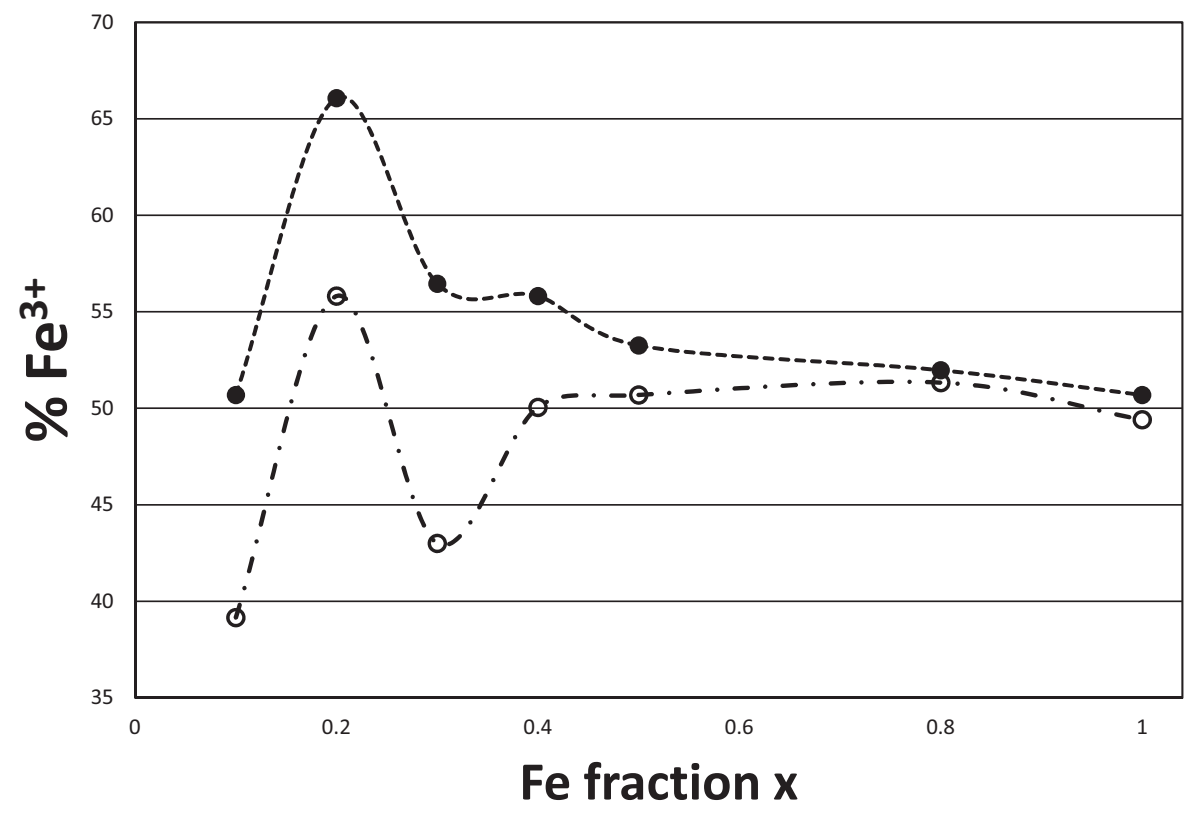

Figure 8: 